\title{
Human Organic Anion Transporters Mediate the Transport of Tetracycline
}

\author{
Ellappan Babu ${ }^{1}$, Michio Takeda ${ }^{1}$, Shinichi Narikawa ${ }^{1}$, Yasuna Kobayashi ${ }^{2}$, Toshinori Yamamoto ${ }^{2}$, \\ Seok Ho Cha ${ }^{1}$, Takashi Sekine ${ }^{1}$, Dhanapal Sakthisekaran ${ }^{3}$ and Hitoshi Endou, ${ }^{1, *}$ \\ ${ }^{1}$ Department of Pharmacology and Toxicology, Kyorin University School of Medicine, \\ 6-20-2 Shinkawa, Mitaka-shi, Tokyo 181-8611, Japan \\ ${ }^{2}$ Department of Clinical Pharmacy, Showa University School of Pharmaceutical Sciences, Tokyo 142-8555, Japan \\ ${ }^{3}$ Department of Medical Biochemistry, University of Madras, Chennai, India
}

Received May 23, 2001 Accepted October 15, 2001

\begin{abstract}
The purpose of this study was to elucidate the molecular mechanism for renal tetracycline transport by human organic anion transporters (hOATs) using proximal tubular cells stably expressing hOATs. The cells stably expressing hOAT1, hOAT2, hOAT3 and hOAT4 exhibited a higher amount of $\left[{ }^{3} \mathrm{H}\right]$ tetracycline uptake compared with mock cells. The apparent $\mathrm{K}_{\mathrm{m}}$ values for hOAT2-, hOAT3- and hOAT4-mediated tetracycline uptakes were $439.9 \pm 23.0,566.2 \pm 28.4$ and $122.7 \pm 16.0 \mu \mathrm{M}$, respectively. Tetracycline significantly inhibited the organic anion uptake by hOAT1, hOAT2 and hOAT4, but not hOAT3. In addition, oxytetracycline, minocycline and doxycycline inhibited the organic anion uptake by hOAT1, whereas oxytetracycline, minocycline but not doxycycline inhibited the organic anion uptake by hOAT2. In contrast, oxytetracycline, minocycline and doxycycline exhibited no significant inhibitory effects on the organic anion uptake by hOAT3 and hOAT4. HOAT1 and hOAT4 mediated the efflux of tetracycline, but hOAT2 and hOAT3 did not. These results suggest that hOAT1, hOAT2 and hOAT3 mediate the basolateral uptake and/or efflux of tetracycline, whereas hOAT4 is responsible for the reabsorption as well as the efflux of tetracycline in the apical side of the proximal tubule. These pharmacological characteristics of hOATs may be significantly related to events associated with the development of tetracycline-induced nephrotoxicity in the human kidney.
\end{abstract}

Keywords: Tetracycline, Organic anion transporter, Proximal tubule, Transport, Cell line

Tetracycline is active against a broad spectrum of bacteria (1). Susceptible strains include a wide range of Gram-positive and Gram-negative bacteria, Mycoplasma, Rickettia and Chlamydia. Tetracyclines are classified as short-acting (tetracycline and oxytetracycline), intermediate-acting (demeclocycline and methacycline) or long-acting (doxycycline and minocycline) based on their serum half-lives of $6-8 \mathrm{~h}, 12 \mathrm{~h}$ or $16-18 \mathrm{~h}$, respectively (2). The main mode of elimination of most tetracycline is via renal glomerular filtration, but it is also eliminated via the biliary route. Approximately $60 \%$ of tetracycline administered is found in the urine in unchanged form (2). However, as far as we know, there has been no report describing the mechanisms of the renal tubular excretion and reabsorption of tetracycline so far.

*Corresponding author. FAX: +81-422-79-1321

E-mail: endouh@kyorin-u.ac.jp
The secretion or reabsorption of numerous organic anions including endogenous metabolites, drugs and xenobiotics, is an important physiological function of the renal proximal tubule (3). Recently, cDNAs encoding renal organic anion transporters (OATs) have been successively cloned. The OATs cloned include OAT1 $(4,5)$, OAT2 (6), OAT3 $(7,8)$, OAT4 (9), OAT-K1 (10), OAT-K2 (11), organic anion-transporting polypeptide (oatp) 1 (12), oatp2 (13), oatp3 (14), multiple resistance-associated protein 2 (MRP2) (15) and human-type I sodium-dependent inorganic phosphate transporter (NPT1) (16).

The purpose of this study was to elucidate the molecular mechanism for renal excretion and reabsorption of tetracycline using $\mathrm{S}_{2}$ cells stably expressing human organic anion transporter (hOAT) 1, hOAT2, hOAT3 and hOAT4 $\left(\mathrm{S}_{2}\right.$ hOAT1, $\mathrm{S}_{2}$ hOAT2, $\mathrm{S}_{2}$ hOAT3 and $\mathrm{S}_{2}$ hOAT4, respectively). Although tetracycline possess no anionic moieties (Fig. 1), we found that tetracycline interacts with hOATs. 
(A)<smiles>CN(C)[C@@H]1C(O)=C(C(N)=O)C(=O)[C@]2(O)C(O)=C3C(=O)c4c(O)cccc4[C@@](C)(O)[C@@H]3C[C@H]12</smiles>

(B)<smiles>CN(C)[C@H]1C(O)=C(C(N)=O)C(=O)[C@]2(O)C(O)=C3C(=O)c4c(O)cccc4[C@@](C)(O)C3(C)C(O)[C@H]12</smiles>

(C)<smiles>CC1c2cccc(O)c2C(=O)C2=C(O)[C@]3(O)C(=O)C(C(N)=O)=C(O)[C@H](N(C)C)[C@H]3[C@H](O)[C@H]21</smiles>

(D)<smiles>CN(C)c1ccc(O)c2c1C[C@H]1C[C@H]3[C@H](N(C)C)C(O)=C(C(N)=O)C(=O)[C@@]3(O)C(O)=C1C2=O</smiles>

Fig. 1. Chemical structure of tetracyclines. A: tetracycline, B: oxyteracycline, C: doxycycline and D: minocycline.

\section{MATERIALS AND METHODS}

\section{Materials}

$\left[{ }^{3} \mathrm{H}\right]$ Tetracycline $(333.6 \mathrm{GBq} / \mathrm{mmol})$ was purchased from Moravek Biochemicals, Inc. (Brea, CA, USA). $\left[{ }^{14} \mathrm{C}\right]$ para-Aminohippuric acid (PAH) $(1.8648 \mathrm{GBq} / \mathrm{mmol})$, $\left[{ }^{3} \mathrm{H}\right]$ estrone sulfate (ES) $(1961 \mathrm{GBq} / \mathrm{mmol})$ and D- $\left[{ }^{14} \mathrm{C}\right] \mathrm{man}-$ nitol $(532.4 \mathrm{GBq} / \mathrm{mmol})$ were purchased from DupontNew England Nuclear (Boston, MA, USA). [ $\left.{ }^{3} \mathrm{H}\right]$ Prostaglandin $\mathrm{F}_{2 \alpha}\left(\mathrm{PGF}_{2 \alpha}\right)(7943.2 \mathrm{GBq} / \mathrm{mmol})$ were purchased from Amersham Pharmacia Biotec. (Buckinghamshire, UK).
Tetracycline, oxytetracycline, minocycline and doxycycline were obtained from Sigma Chemical Co. (St. Louis, MO, USA). Fetal bovine serum (FBS), trypsin and geneticin were obtained from Gibco/BRL (Gaithersburg, MD, USA), recombinant epidermal growth factor (EGF) from Wakunaga (Hiroshima), insulin from Shimizu (Shizuoka), RITC 80-7 culture medium from Iwaki Co. (Tokyo) and TfX-50 from Promega (Madison, WI, USA).

Cell culture and establishment of $S_{2}$ hOAT2 and $S_{2}$ hOAT4

$\mathrm{S}_{2}$ cells, derived from transgenic mice harboring the temperature-sensitive simian virus 40 large $\mathrm{T}$-antigen gene, were established as described previously (17). The establishment and characterization of $\mathrm{S}_{2}$ hOAT1 and $\mathrm{S}_{2}$ hOAT3 have been reported previously (18). The full-length cDNA of hOAT2 was isolated by screening human kidney cDNA library using rat OAT2 cDNA (6) as the probe. The fulllength cDNAs of hOAT2 and hOAT4 (8) were subcloned into pcDNA 3.1 (Invitrogen, San Diego, CA, USA), a mammalian expression vector. $\mathrm{S}_{2}$ hOAT2 and $\mathrm{S}_{2}$ hOAT4 were obtained by transfecting $\mathrm{S}_{2}$ cells with pcDNA3.1hOAT2 or pcDNA3.1-hOAT4 coupled with $\mathrm{pSV}_{2}$ neo, a neomycin resistance gene using a liposome (19). $\mathrm{S}_{2}$ cells transfected with pcDNA3.1 lacking an insert and $\mathrm{pSV}_{2}$ neo were designated as $\mathrm{S}_{2}$ pcDNA 3.1 and used as a control (mock cells). These cells were grown in a humidified incubator at $33^{\circ} \mathrm{C}$ and $5 \% \mathrm{CO}_{2}$, using RITC $80-7$ medium (Iwaki Co.) that contained $5 \%$ of FBS, $10 \mu \mathrm{g} / \mathrm{ml}$ of transferrin, $0.08 \mathrm{U} / \mathrm{ml}$ of insulin, $10 \mathrm{ng} / \mathrm{ml}$ of recombinant EGF and $400 \mu \mathrm{g} / \mathrm{ml}$ of geneticin (19). The cells were subcultured in a medium containing $0.05 \%$ trypsin-EDTA solution $(137 \mathrm{mM} \mathrm{NaCl}, 5.4 \mathrm{mM} \mathrm{KCl}, 5.5 \mathrm{mM}$ glucose, $4 \mathrm{mM} \mathrm{NaHCO} 3,0.5 \mathrm{mM}$ EDTA and $5 \mathrm{mM}$ HEPES; $\mathrm{pH}$ 7.2) and used for $25-35$ passages. Clonal cells were isolated using a cloning cylinder and screened by determining the optimal substrate for each transporter; i.e., $\left[{ }^{14} \mathrm{C}\right] \mathrm{PAH}$ for hOAT1 (5), $\left[{ }^{3} \mathrm{H}\right] \mathrm{PGF}_{2 \alpha}$ for hOAT2 and $\left[{ }^{3} \mathrm{H}\right]$ ES for hOAT3 and hOAT4 $(8,9) . \mathrm{S}_{2}$ hOAT2 and $\mathrm{S}_{2}$ hOAT4 exhibited a dose-and time-dependent increase in $\mathrm{PGF}_{2 \alpha}$ and ES uptake activities, respectively. The $\mathrm{S}_{2}$ monolayer was determined to be leaky based on the results of a study wherein we estimated paracellular secretion from cells cultured on a permeable support, using D- $\left[{ }^{14} \mathrm{C}\right] \mathrm{man}-$ nitol as an indicator. In addition, vertical sections of $\mathrm{S}_{2}$ hOAT2 and $\mathrm{S}_{2}$ hOAT4 stained with polyclonal antibodies against hOAT2 and hOAT4, respectively, showed that the subcellular localization of hOAT2 and hOAT4 proteins was mainly on the cell membrane (A. Enomoto et al., unpublished observation; E. Babu et al., unpublished observation). Both the basolateral and apical portions of the membrane showed positive staining. Therefore, the cells were cultured on a solid support for the following experiments. 


\section{Uptake experiment}

Uptake experiments were performed as previously described $(18,19)$. The $\mathrm{S}_{2}$ cells were seeded in 24-well tissue culture plates at a density of $1 \times 10^{5}$ cells/well. After the cells were cultured for two days, the cells were washed three times with Dulbecco's modified phosphate-buffered saline (D-PBS) solution ( $137 \mathrm{mM} \mathrm{NaCl}, 3 \mathrm{mM} \mathrm{KCl}, 8 \mathrm{mM}$ $\mathrm{NaHPO}_{4}, 1 \mathrm{mM} \mathrm{KH} \mathrm{PO}_{4}, 1 \mathrm{mM} \mathrm{CaCl} 2$ and $0.5 \mathrm{mM} \mathrm{MgCl}_{2}$, $\mathrm{pH}$ 7.4) containing $5 \mathrm{mM}$ D-glucose and then preincubated with the same solution at $37^{\circ} \mathrm{C}$ for $10 \mathrm{~min}$ in a water bath. The cells were then incubated in D-PBS containing $5 \mathrm{mM}$ D-glucose with $\left[{ }^{3} \mathrm{H}\right]$ tetracycline at $37^{\circ} \mathrm{C}$, as indicated in each experiment. The uptake was stopped by the addition of ice-cold D-PBS, and the cells were washed three times with the same solution. The cells in each well were lysed with $0.5 \mathrm{ml}$ of $0.1 \mathrm{~N}$ sodium hydroxide and $2.5 \mathrm{ml}$ of aquasol-2, and radioactivity was determined using a $\beta$ scintillation counter (LSC-3100; Aloka, Tokyo).

\section{Inhibition study}

To evaluate the inhibitory effects of tetracyclines on the organic anion transport by hOATs, $\mathrm{S}_{2}$ hOAT1, $\mathrm{S}_{2}$ hOAT2,
$\mathrm{S}_{2}$ hOAT3 and $\mathrm{S}_{2}$ hOAT4 were incubated in solution containing either $5 \mu \mathrm{M}\left[{ }^{14} \mathrm{C}\right] \mathrm{PAH}$ for $2 \mathrm{~min}$ (hOAT1), $50 \mathrm{nM}$ $\left[{ }^{3} \mathrm{H}\right] \mathrm{PGF}_{2 \alpha}$ for $30 \mathrm{~s}(\mathrm{hOAT} 2)$ or $50 \mathrm{nM}\left[{ }^{3} \mathrm{H}\right] \mathrm{ES}$ for $2 \mathrm{~min}$ (hOAT3 and hOAT4) in the absence or presence of tetracyclines. Tetracycline, oxytetracycline, minocycline and doxycycline were dissolved in distilled $\mathrm{H}_{2} \mathrm{O}$ and diluted with the incubation medium.

\section{Efflux study}

The efflux study was performed as previously described (19). $\mathrm{S}_{2}$ hOAT1, $\mathrm{S}_{2}$ hOAT2, $\mathrm{S}_{2}$ hOAT3, $\mathrm{S}_{2}$ hOAT4 and mock cells were seeded in 24-well tissue culture plates at a density of $1 \times 10^{5}$ cells/well. After the cells were cultured for two days, the cells were washed three times with D-PBS and then preincubated in the same solution at $37^{\circ} \mathrm{C}$ for $10 \mathrm{~min}$ in a water bath. Thereafter, the monolayers were incubated with $2 \mu \mathrm{M}\left[{ }^{3} \mathrm{H}\right]$ tetracycline at $37^{\circ} \mathrm{C}$ for $30 \mathrm{~min}$, washed immediately three times with D-PBS and then incubated with $500 \mu 1$ of D-PBS. After the incubation for the indicated periods of time, $50 \mu 1$ of the supernatant was collected. After the incubation, the medium was aspirated immediately and the cell monolayers were
(A)

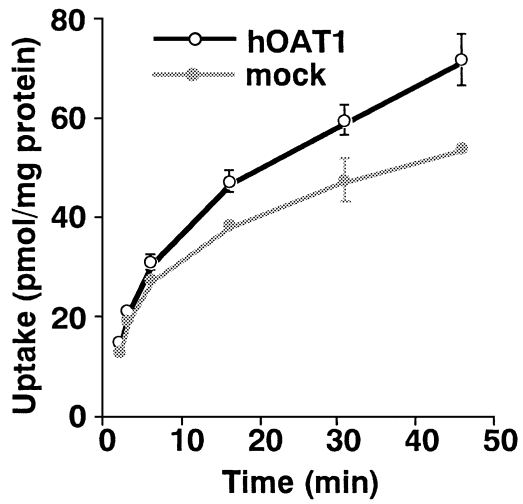

(B)

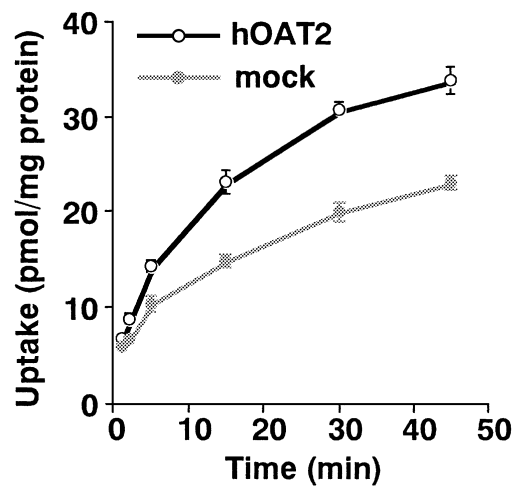

(C)

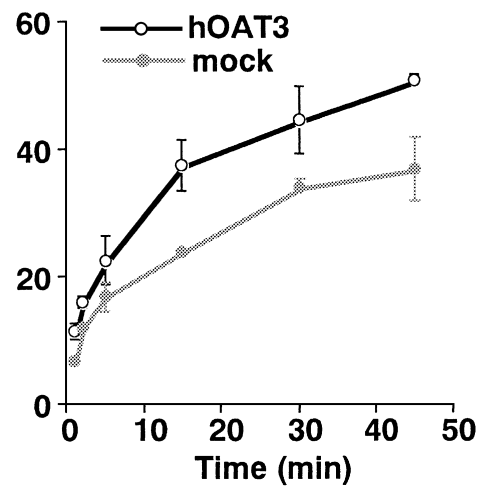

(D)

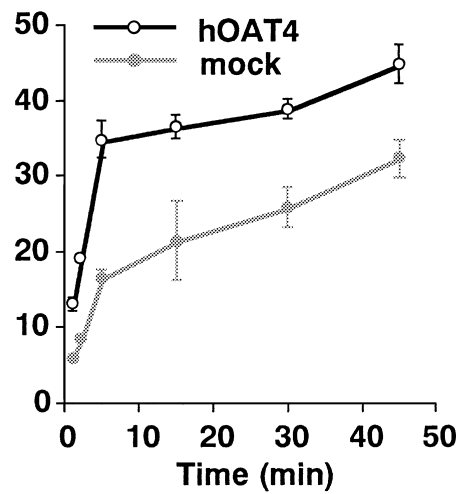

Fig. 2. Time course of tetracycline uptake by hOATs. $\mathrm{S}_{2}$ hOAT1 (A), $\mathrm{S}_{2}$ hOAT2 (B), $\mathrm{S}_{2}$ hOAT3 (C), $\mathrm{S}_{2}$ hOAT4 (D) and $\mathrm{S}_{2}$ pcDNA 3.1 (mock cells) were incubated in solution containing $2 \mu \mathrm{M}\left[{ }^{3} \mathrm{H}\right]$ tetracycline for $1 \mathrm{~min}$ to $45 \mathrm{~min}$ at $37^{\circ} \mathrm{C}$. The values of the mock cells were subtracted. Each value represents the mean \pm S.E.M. of four determinations. 


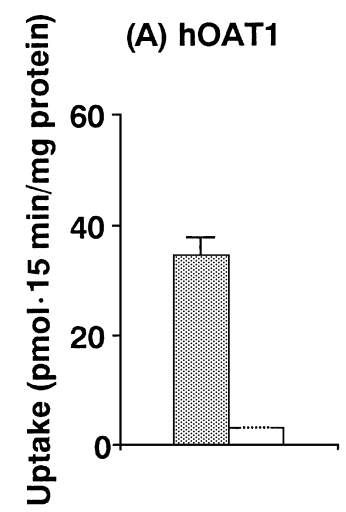
(B) hOAT2

(C) hOAT3

(D) hOAT4

(E) Mock
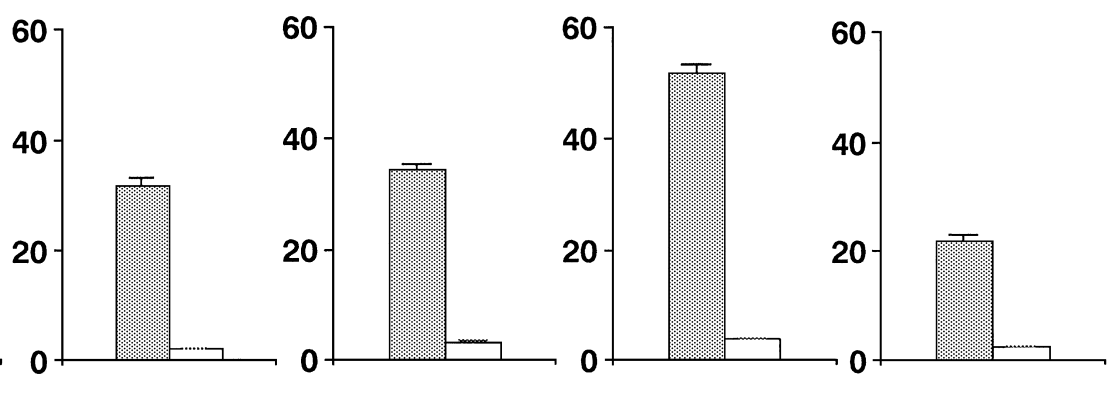

Fig. 3. Specificity of tetracycline uptake by hOATs. $\mathrm{S}_{2}$ hOAT1 (A), $\mathrm{S}_{2}$ hOAT2 (B), $\mathrm{S}_{2}$ hOAT3 (C), $\mathrm{S}_{2}$ hOAT4 (D) and $\mathrm{S}_{2}$ pcDNA 3.1 (mock cells) (E) were incubated in solution containing $2 \mu \mathrm{M}\left[{ }^{3} \mathrm{H}\right]$ tetracycline and $2 \mu \mathrm{M}\left[{ }^{14} \mathrm{C}\right]$ mannitol for 15 min at $37^{\circ} \mathrm{C}$. Each value represents the mean \pm S.E.M. of four determinations.

(A) hOAT2

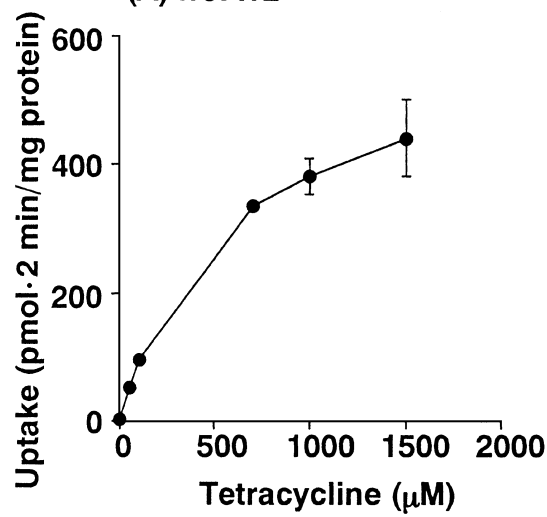

(D) hOAT2

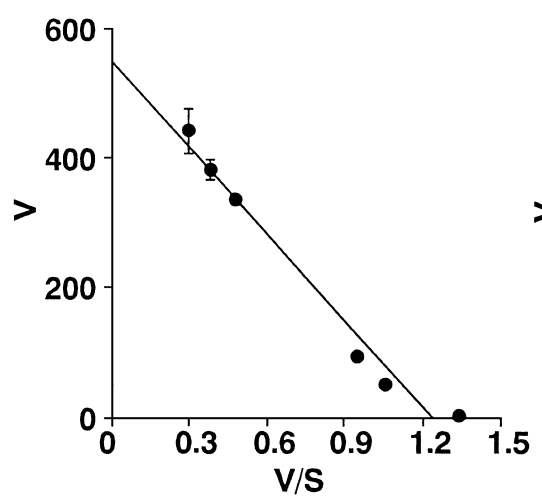

(B) hOAT3

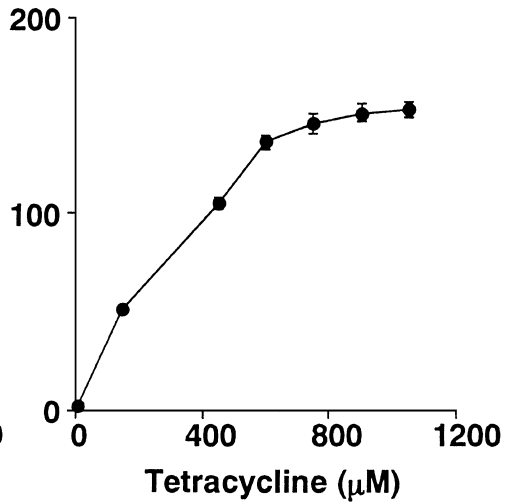

(E) hOAT3

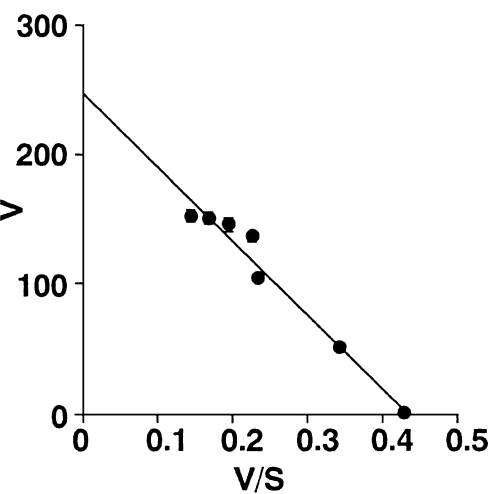

(C) hOAT4

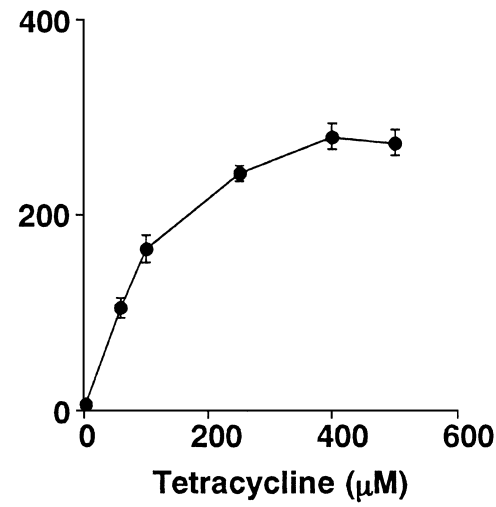

(F) hOAT4

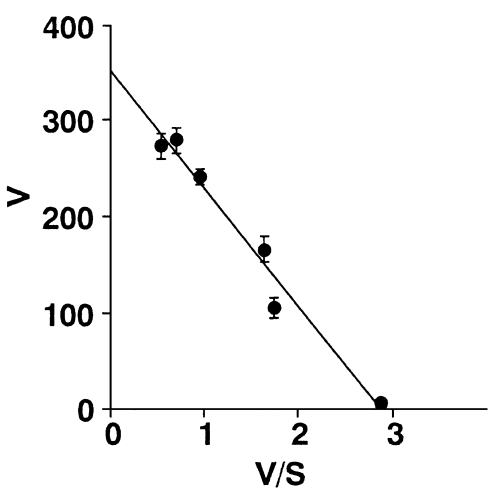

Fig. 4. Concentration dependence of tetracycline uptake by hOAT2 (A), hOAT3 (B) and hOAT4 (C) and its kinetic analysis. $\mathrm{S}_{2}$ hOAT2, $\mathrm{S}_{2}$ hOAT3, $\mathrm{S}_{2}$ hOAT4 and $\mathrm{S}_{2}$ pcDNA 3.1 (mock cells) were incubated in solution containing various concentrations of $\left[{ }^{3} \mathrm{H}\right]$ tetracycline for $2 \mathrm{~min}$ at $37^{\circ} \mathrm{C}$. The values of the mock cells were subtracted. The Eadie-Hofstee plots of the uptake of $\left[{ }^{3} \mathrm{H}\right]$ tetracycline by hOAT2 (D), hOAT3 (E) and hOAT4 (F) were analyzed. Each value represents the mean \pm S.E.M. of four determinations. 
washed three times with the medium and solubilized in $0.5 \mathrm{ml}$ of $0.1 \mathrm{~N}$ sodium hydroxide. The radioactivities within the supernatant and cell lysate were measured by counting the radioactivity. The rate of efflux at each time point was calculated by the following formula: (effluxed $\left[{ }^{3} \mathrm{H}\right]$ tetracycline by transfectants - effluxed $\left[{ }^{3} \mathrm{H}\right]$ tetracycline by mock $) /\left(\left[{ }^{3} \mathrm{H}\right]\right.$ tetracycline accumulated by transfectants at time $0-\left[{ }^{3} \mathrm{H}\right]$ tetracycline accumulated by mock at time 0 ).
Statistical analyses

Data are expressed as the mean \pm S.E.M. Statistical differences were determined by Student's unpaired $t$-test. Differences were considered significant at $P<0.05$.

\section{RESULTS}

Tetracycline uptake by hOATs

We evaluated the time-dependent uptake of tetracycline by hOATs. As shown in Fig. 2, $\mathrm{S}_{2}$ hOAT1 (A), $\mathrm{S}_{2}$ hOAT2
(A) hOAT1

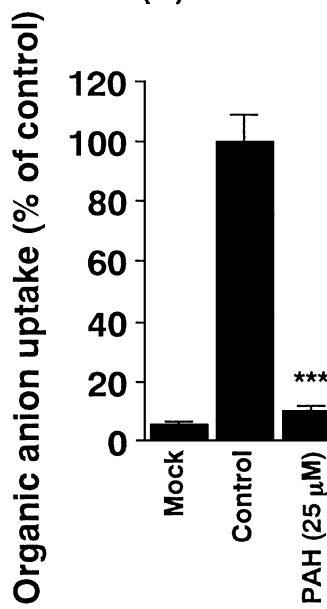

(B) hOAT2

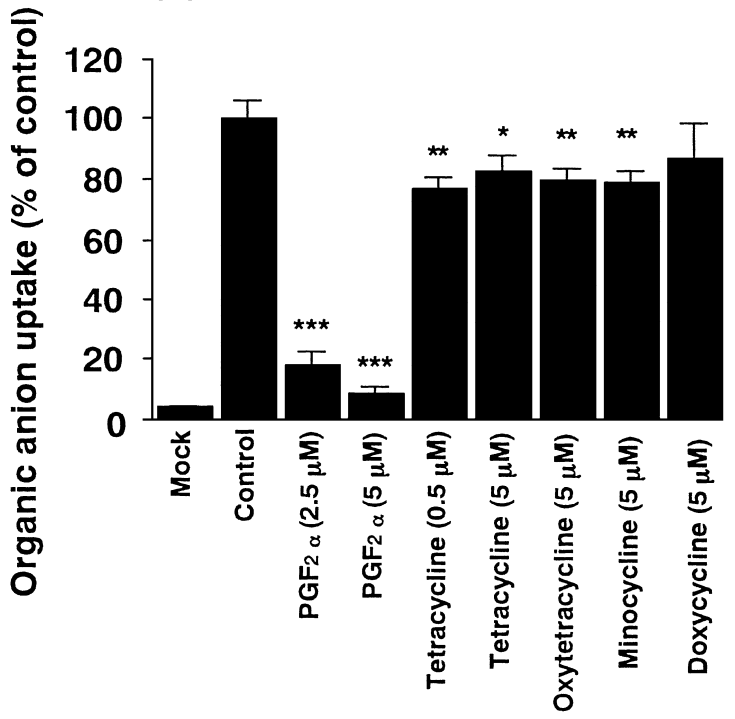

(C) hOAT3

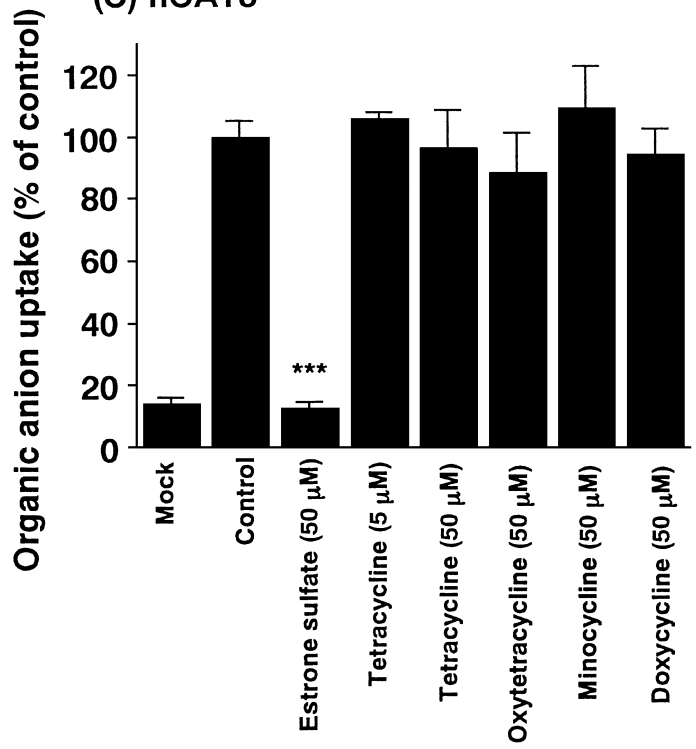

(D) hOAT4

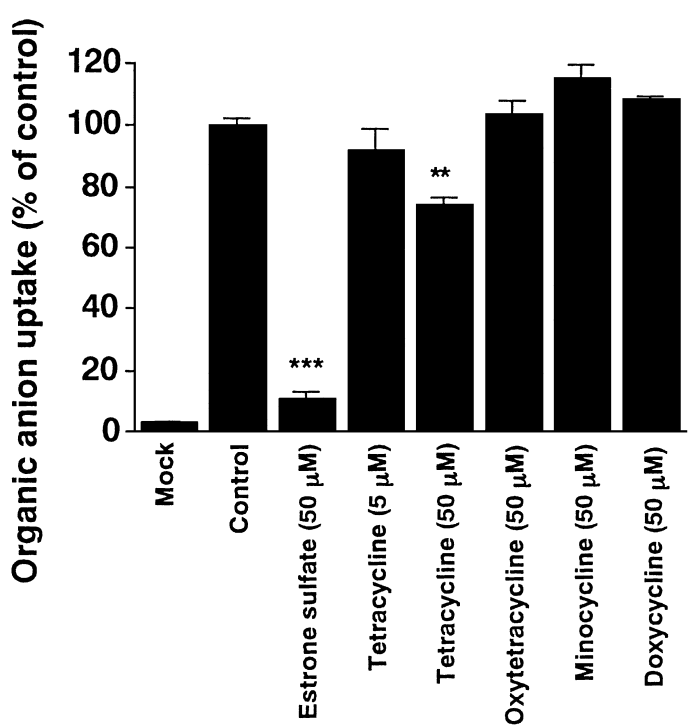

Fig. 5. Effects of tetracyclines on the organic anion uptake by hOATs. $\mathrm{S}_{2}$ hOAT1 (A), $\mathrm{S}_{2}$ hOAT2 (B), $\mathrm{S}_{2}$ hOAT3 (C) and $\mathrm{S}_{2}$ hOAT4 (D) were incubated in solution containing either $5 \mu \mathrm{M}\left[{ }^{14} \mathrm{C}\right] \mathrm{PAH}$ for $2 \mathrm{~min}$ (hOAT1), $5 \mathrm{nM}\left[{ }^{3} \mathrm{H}\right] \mathrm{PGF}_{2 \alpha}$ for $30 \mathrm{~s}$ (hOAT2) or $50 \mathrm{nM}\left[{ }^{3} \mathrm{H}\right] \mathrm{ES}$ for $2 \mathrm{~min}$ (hOAT3 and hOAT4) in the absence or presence of tetracycline, oxytetracycline, minocycline or doxycycline for $2 \mathrm{~min}$ at $37^{\circ} \mathrm{C}$. Each value represents the mean \pm S.E.M. of four determinations. ${ }^{*} P<0.05,{ }^{* *} P<0.01$ and $* * * P<0.001$ vs control. 
(B), $\mathrm{S}_{2}$ hOAT3 (C) and $\mathrm{S}_{2}$ hOAT4 (D) exhibited higher amounts of tetracycline uptake than $\mathrm{S}_{2}$ pcDNA 3.1. In order to confirm the specificity of tetracycline uptake mediated by hOATs, cells were incubated in a solution containing $2 \mu \mathrm{M}\left[{ }^{3} \mathrm{H}\right]$ tetracycline and $2 \mu \mathrm{M}\left[{ }^{14} \mathrm{C}\right]$ mannitol for $15 \mathrm{~min}$. As shown in Fig. 3, tetracycline uptake values by these transfectants were higher than that by the mock cells, whereas mannitol uptake values were comparable among these cells.

The kinetics of tetracycline uptake was examined to evaluate the pharmacological characteristics of hOAT2, hOAT3 and hOAT4 on the tetracycline uptake. Since the amount of specific tetracycline uptakes in $\mathrm{S}_{2}$ hOAT1 was small, kinetic analysis of the concentration dependence was not performed for hOTA1. The specific uptake of tetracycline by hOAT2, hOAT3 and hOAT4 revealed saturable kinetics (Fig. 4: A, B and C), and the Eadie-Hofstee plots gave a single straight line (Fig. 4: D, E and F). The estimated $\mathrm{K}_{\mathrm{m}}$ values of tetracycline uptake by hOAT2, hOAT3 and hOAT4 were $439.9 \pm 23.0 \mu \mathrm{M}(\mathrm{N}=4), 566.2 \pm 28.4 \mu \mathrm{M}$ $(\mathrm{N}=4)$ and $122.7 \pm 16.0 \mu \mathrm{M}(\mathrm{N}=4)$, respectively. The results suggest that hOAT1, hOAT2, hOAT3 and hOAT4 are responsible for the transport of tetracycline.

\section{Inhibitory effects of tetracyclines on the organic anion} uptake by hOATs

In order to elucidate whether hOATs interact with tetracyclines, we examined the effects of various tetracyclines on the organic anion uptake by hOAT1, hOAT2, hOAT3 and hOAT4. As shown in Fig. 5A, tetracycline, oxyteracycline, minocycline and doxycycline significantly inhibited the organic anion uptake by hOAT1 $(\mathrm{N}=4, P<0.001$ and $P<0.01$ vs control). As shown in Fig. $5 \mathrm{~B}$, tetracycline, oxyteracycline and minocycline $(\mathrm{N}=4, P<0.01$ and $P<0.05$ vs control), but not doxycycline, significantly inhibited the organic anion uptake by hOAT2. In contrast, none of the tetracyclines tested inhibited the hOAT3-mediated organic anion uptake $(\mathrm{N}=4$, N.S. vs control) (Fig. 5C). Figure 5D shows that tetracycline $(\mathrm{N}=4, P<0.01$ vs control), but not oxytetracycline, minocycline and doxycycline, significantly inhibited the organic anion uptake by hOAT4.

\section{Efflux of tetracycline by hOATs}

In order to determine whether hOATs mediate the efflux of tetracycline, we compared the efflux of tetracycline by $\mathrm{S}_{2}$ hOAT1, $\mathrm{S}_{2}$ hOAT2, $\mathrm{S}_{2}$ hOAT3 and $\mathrm{S}_{2}$ hOAT4 with that by mock cells. As shown in Fig. 6, $\mathrm{S}_{2}$ hOAT1 (A) and $\mathrm{S}_{2}$ hOAT4 (B) exhibited a significant amount of tetracycline efflux, in which the amount of tetracycline efflux by mock cells was subtracted. In contrast, $\mathrm{S}_{2}$ hOAT2 and $\mathrm{S}_{2}$ hOAT3 did not mediate tetracycline efflux (data not shown).
(A) hOAT1

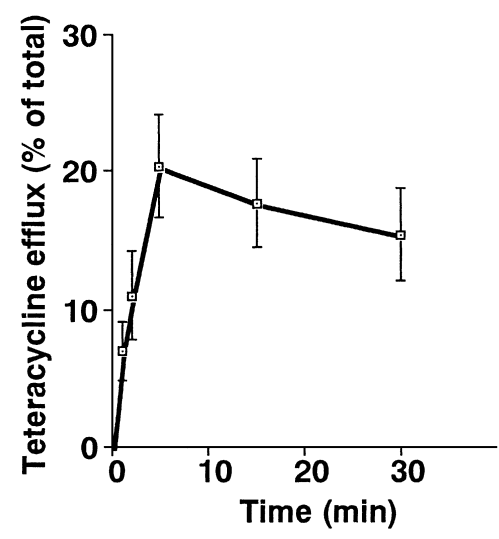

(B) hOAT4

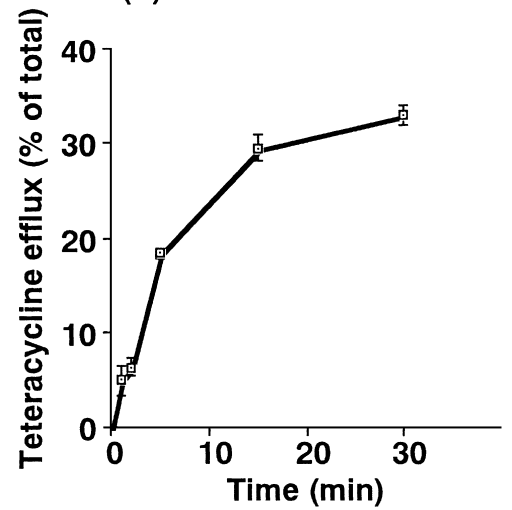

Fig. 6. Efflux of tetracycline from $\mathrm{S}_{2}$ hOAT1 and $\mathrm{S}_{2}$ hOAT4. After 30 -min incubation at $37^{\circ} \mathrm{C}$ with $2 \mu \mathrm{M}\left[{ }^{3} \mathrm{H}\right]$ tetracycline, $\mathrm{S}_{2}$ hOAT1, $\mathrm{S}_{2}$ hOAT4 and $\mathrm{S}_{2}$ pcDNA 3.1 (mock cells) were incubated at $37^{\circ} \mathrm{C}$ for $30 \mathrm{~min}$. The rate of efflux at each time point was calculated by the following formula: (effluxed $\left[{ }^{3} \mathrm{H}\right]$ tetracycline by the transfectants effluxed $\left[{ }^{3} \mathrm{H}\right]$ tetracycline by mock $) /\left(\left[{ }^{3} \mathrm{H}\right]\right.$ tetracycline accumulated by the transfectants at time $0-\left[{ }^{3} \mathrm{H}\right]$ tetracycline accumulated by mock at time 0 ). Each value represents the mean \pm S.E.M. of three determinations.

\section{DISCUSSION}

hOAT1 and hOAT3 have been shown to mediate the transport of nonsteroidal anti-inflammatory drugs, antitumor drugs, histamine $\mathrm{H}_{2}$-receptor antagonist, prostaglandins, diuretics, angiotensin-converting enzyme inhibitors and beta-lactam antibiotics $(5,9)$. Some differences in characteristics exist between hOAT1 and hOAT3, such as substrate specificity and localization: hOAT1 at the basolateral side of the $S_{2}$ segment of the proximal tubule (5) versus hOAT3 at the first, second and third segments $\left(\mathrm{S}_{1}, \mathrm{~S}_{2}\right.$ and $\mathrm{S}_{3}$ ) of the proximal tubule (9). In addition, hOAT1, but not hOAT3, exhibits transport properties as an exchanger $(5,9)$. hOAT2, also identified to be localized to the basolateral side of the proximal tubule, mediated the transport of antivirals, including zidovudine and acyclovir, and sali- 
cylate, but not para-aminohippuric acid, estrone sulfate, methotrexate and ochratoxin A (Y. Kobayashi et al., unpublished observation). HOAT4 was isolated from human placenta (8) and found to localize to the apical side of the proximal tubule in the kidney. HOAT4 was shown to mediate the transport of various anionic drugs including para-aminohippuric acid, estrone sulfate, methotrexate and ochratoxin A (ref. 8; E. Babu et al., unpublished observation).

In the current study, hOAT1, hOAT2, hOAT3 and hOAT4 were shown to mediate the uptake of tetracycline. In addition, tetracycline significantly inhibited the organic anion uptake by hOAT1, hOAT2 and hOAT4, which confirmed the interaction between tetracycline and hOAT1, hOAT2 or hOAT4. Thus, it was suggested that hOAT1, hOAT2 and hOAT3 mediate the uptake of tetracycline in the basolateral side of the proximal tubule, whereas hOAT4 mediated not only the reabsorption but also the efflux of tetracycline in the apical side of the proximal tubule. Renal clearance of tetracycline in normal subjects was reported to be $74 \mathrm{ml} / \mathrm{min}(20)$, whereas the creatinine clearance was $104-139 \mathrm{ml} / \mathrm{min}(21)$. Thus, it was suggested that urinary tetracycline is reabsorbed in tubules, and hOAT4 plays some roles in this reabsorption. Tetracycline exhibited no significant inhibitory effects on hOAT3-mediated organic anion uptake. The results were inconsistent with the tetracycline uptake activity shown in Figs. 2, 3 and 4. The reason for this discrepancy remains unknown and the further studies should be performed to clarify this.

The results of the inhibition experiments provide the possibility that oxytetracycline, minocycline and doxycycline are taken up via hOAT1 and hOAT2 on the basolateral side of the proximal tubule. In contrast, since oxytetracycline, minocycline and doxycycline exhibited no significant inhibitory effects on hOAT4-mediated organic anion uptake, it should be elucidated whether apical transporters mediating organic anion transport other than hOAT4, i.e., OAT-K1 (10), OAT-K2 (11), oatp1 (12), MRP2 (15) and NPT1 (16), interact with these tetracyclines. In addition to these specific pathways, since minocycline and doxycycline are lipophilic, these drugs are also transported via passive diffusion. In addition, the molecular background of these divergent inhibitory effects of tetracyclines on hOATs should be elucidated in terms of the structure-function relationship.

Tetracyclines have been shown to induce various forms of nephrotoxicity $(1,22)$. Outdated tetracycline products resulted in a Fanconi-like syndrome (renal tubular abnormality), with acidosis, nephrosis and aminoaciduria. Tetracyclines may also cause further increases in blood urea nitrogen and serum creatinine levels in patients with renal failure. These biochemical changes as well as tetracycline-induced azotemia have been attributed to the anti- anabolic effect of the drug. One possible explanation of how hOATs are associated with tetracycline-induced nephrotoxicity is that hOAT1, hOAT2 and hOAT3 mediate tetracycline uptake in the basolateral side, while hOAT4 mediates tetracycline reabsorption in the apical side, which may lead to the accumulation of tetracycline and the induction of its nephrotoxic effects. In contrast, toxicological implication of hOAT4-mediated tetracycline efflux remains unknown and further studies should be performed.

In conclusion, these results suggest that hOATs mediate the urinary excretion and the reabsorption of tetracycline in the proximal tubule. These transport characteristics may be associated with the induction of tetracycline-induced nephrotoxicity.

\section{Acknowledgments}

This study was supported in part by Grants-in-Aid from the Ministry of Education, Culture, Sports, Science and Technology (No. 11671048, No. 11694310 and No. 13671128), the Science Research Promotion Fund of the Japan Private School Promotion Foundation, and Research on Health Sciences focusing on Drug Innovation from the Japan Health Sciences Foundation.

\section{REFERENCES}

1 Uetrecht $\mathrm{J}$ and Walmsley SL: Antimicrobial agents that affect the synthesis of cellular proteins. In Principles of Medical Pharmacology (6th ed), Edited by Kalant $\mathrm{H}$ and Roschlau WHE, pp 656 - 676, Oxford University Press, New York (1992)

2 Chambers HF: Chloramphenicol, tetracyclines, macrolides, clindamycin \& streptogramins. In Basic \& Clinical Pharmacology (8th ed), Edited by Katzung BG, pp 774-783, McGraw-Hill, New York (2001)

3 Pritchard JB and Miller DS: Mechanisms mediating renal secretion of organic anions and cations. Physiol Rev 73, 765-796 (1993)

4 Sekine T, Watanabe N, Hosoyamada M, Kanai Y and Endou H: Expression cloning and characterization of of a novel multispecific organic anion transporter. J Biol Chem 272, 18526 18529 (1997)

5 Hosoyamada M, Sekine T, Kanai Y and Endou H: Molecular cloning and functional expression of a multispecific organic anion transporter from human kidney. Am J Physiol 276, F122 F128 (1999)

6 Sekine T, Cha SH, Tsuda M, Apiwattanakul N, Nakajima N, Kanai $\mathrm{Y}$ and Endou $\mathrm{H}$ : Identification of multispecific organic anion transporter 2 expressed predominantly in the liver. FEBS Lett 429, 179 - 182 (1998)

7 Kusuhara H, Sekine T, Utsunomiya-Tate N, Tsuda M, Kojima R, Cha SH, Sugiyama Y, Kanai Y and Endou H: Molecular cloning and characterization of a new multispecific organic anion transporter from rat brain. J Biol Chem 274, 13675 - 13680 (1999)

8 Cha SH, Sekine T, Kusuhara H, Yu E, Kim YJ, Kim DK, Sugiyama $\mathrm{Y}$, Kanai $\mathrm{Y}$ and Endou $\mathrm{H}$ : Molecular cloning and characterization of multispecific organic anion transporter 4 expressed in the placenta. J Biol Chem 275, 4507-4512 (2000)

9 Cha SH, Sekine T, Fukushima JI, Kanai Y, Kobayashi Y, Goya 
$\mathrm{T}$ and Endou $\mathrm{H}$ : Identification and characterization of human organic anion transporter 3 expressing predominantly in the kidney. Mol Pharmacol 59, 1277 - 1286 (2001)

10 Saito H, Masuda S and Inui KI: Cloning and functional characterization of a novel rat organic anion transporter mediating basolateral uptake of methotrexate in the kidney. J Biol Chem 271, 20719 - 20725 (1996)

11 Masuda S, Ibaramoto K, Takeuchi A, Saito H, Hashimoto Y and Inui KI: Cloning and functional characterization of a new multispecific organic anion transporter, OAT-K2, in rat kidney. Mol Pharmacol 55, 743 - 752 (1999)

12 Jacquemin E, Hagenbuch B, Stieger B, Wolkoff AW and Meier PJ: Expression cloning of a rat liver $\mathrm{Na}^{+}$-independent organic anion transporter. Proc Natl Acad Sci USA 91, 133 - 137 (1994)

13 Noe B, Hagenbuch B, Stieger B and Meier PJ: Isolation of a multispecific organic anion and cardiac glycoside transporter from rat brain. Proc Natl Acad Sci USA 94, 10346-10350 (1997)

14 Abe T, Kakyo M, Sakagami B, Tokui T, Nishio T, Tanemoto M, Nomura H, Hebert SC, Matsuno S, Kondo $\mathrm{H}$ and Yawo $\mathrm{H}$ : Molecular characterization and tissue distribution of a new organic anion transporter subtype (oatp3) that transports thyroid hormones and taurocholate and comparison with oatp2. J Biol Chem 273, 22395 - 22401 (1998)

15 Leier I, Hummel-Eisenbeiss J, Cui Y and Keppler D: ATPdependent para-aminohippurate transport by apical multidrug resistance protein MRP2. Kidney Int 57, 1636 - 1642 (2000)
16 Uchino H, Tamai I, Yamashita K, Minemoto Y, Sai Y, Yabuuchi $\mathrm{H}$, Miyamoto KI, Takeda E and Tsuji A: p-Aminohippuric acid transport at renal apical membrane mediated by human inorganic phosphate transporter NPT1. Biochem Biophys Res Commun 270, 254-259 (2000)

17 Hosoyamada M, Obinata M, Suzuki M and Endou H: Cisplatininduced toxicity in immortalized renal cell lines established from SV40 large T-antigen gene. Arch Toxicol 70, 284 - 292 (1996)

18 Takeda M, Hosoyamada M, Cha SH, Sekine T and Endou H: Hydrogen peroxide downregulates human organic anion transporters in the basolateral side of the proximal tubule. Life Sci 68, 679- $687(2000)$

19 Takeda M, Tojo A, Sekine T, Hosoyamada M, Kanai Y and Endou H: Role of organic anion transporter 1 (OAT1) in cephaloridine (CER)-induced nephrotoxicity. Kidney Int 56, $2128-2136$ (1999)

20 Aaggard GN: Tetracyclines. In AMA drug evaluations 5 th ed, Edited by Bennet DR, pp $1667-1680$, American Medical Association, Chicago (1983)

21 Gabbai FB and Blantz RC: Glomerular filtration. In Textbook of Nephrology, 4th ed, Edited by Massry SG and Glassock RJ, pp 56-64, Lippincott Williams \& Wilkins, Philadelphia (2001)

22 Bihorac A, Ozener C, Akoglu E and Kullu S: Tetracyclineinduced acute interstitial nephritis as a cause of acute renal failure. Nephron 81, 72 - 75 (1999) 